\title{
Ischaemic stroke among young people aged 15 to 50 years in Adelaide, South Australia
}

Matthew C L Phillips MB BS, Neurology Registrar

James M Leyden MB BS, FRACP, Neurologist

Woon K Chon MB BS, FRANZCR Radiologist

Tim Kleinig MB BS(Hons), PhD, FRACP, Neurologist

Philippa Czapran MB BS, Medical Student Neurology

Andrew Lee MB BS, FRACP, Director of Stroke Medicine

Simon A Koblar BM BS, FRACP, PhD Associate Professor

Jim Jannes MB BS, FRACP, PhD, Head Stroke Unit

1 Queen Elizabeth Hospital Adelaide, SA.

2 Royal Adelaide Hospital, Adelaide, SA

3 Comprehensive Strok Centre, Flinders Medica Centre, Adelaide, SA.

4 Stroke Research Programme, University of Adelaide, Adelaide, SA

james.leyden@ health.sa.gov.au

MJA 2011; 195: 610-614 doi: 10.5694/mjall.10558

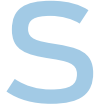

troke can have disastrous consequences for the individual and society; it is Australia's second leading cause of death. ${ }^{1}$ The national lifetime cost for all first-ever strokes occurring in Australia in 1997 was estimated to be $\$ 1.3$ billion. $^{2}$ Strokes among young people account for $18 \%$ of all strokes in Australia and are particularly damaging, as patients are often unable to resume independent living or return to the workforce. ${ }^{1}$

Population-specific studies are essential to our understanding of stroke risk, as considerable differences in risk factors and aetiologies can exist across different countries and ethnic groups. To date, aetiological studies of ischaemic stroke among young people have been reported from several countries in Europe, the Americas and Asia $^{3-17}$ but, to our knowledge, none have been reported for the Australian population. Furthermore, only a minority of studies elsewhere have presented detailed information regarding less well documented stroke risk factors and neuroimaging features. ${ }^{3,7,17}$

The purpose of this study was to determine risk factors, aetiologies, and neuroimaging features among young Australian people who have had a first-ever ischaemic stroke, and to evaluate these with regard to age, sex and ethnicity. We aimed to be able to compare our findings with those for other countries.

\section{Methods}

South Australia is a state of 1.6 million people. Adelaide, the largest city and capital, has a population of 1.2 million people and is the only major referral centre for stroke in the state. Our retrospective study included all seven public hospitals in the greater Adelaide area. Formal ethics approval was sought and obtained from each hospital ethics board.

The public hospital coding system was searched for all patients aged 15

\section{Abstract}

Objectives: To report risk factors, aetiology and neuroimaging features among a large series of young Australian patients who were admitted to hospital for a first-ever occurrence of ischaemic stroke; to analyse the effect of age, sex and ethnicity on the presence of risk factors; and to compare Australian and overseas data.

Design, setting and patients: Retrospective evaluation of data for all patients aged from 15 to 50 years who were admitted to a public hospital in Adelaide, South Australia, from January 2006 to June 2010 with a primary diagnosis of ischaemic stroke.

Results: Among 326 patients (184 males), the most frequent stroke risk factors overall were dyslipidaemia (187), smoking (161), hypertension (105) and obesity (92). Fifty-one patients used illicit drugs, mostly comprising marijuana and amphetamines. The most frequent stroke aetiologies overall were cardioembolism (85), arterial dissection (49), and small-vessel occlusion (31). Cardioembolism was highly prevalent among our study population compared with patients in other countries. Neuroimaging showed that more patients in our study had strokes that involved both vascular territories concurrently (9\%) compared with patients in other countries.

Conclusions: Risk factors, aetiology and features of ischaemic stroke among young people in Adelaide differ significantly from published data for young patients around the world. Patients in Adelaide are more likely to be obese, to be misusing marijuana and amphetamines, to suffer a cardioembolic event and to have a stroke that concurrently affects both the anterior and posterior cerebral circulation.

to 50 years with a primary diagnosis of any form of stroke who were admitted to a public hospital in Adelaide from January 2006 to June 2010. We reviewed discharge summaries, neuroimaging results and medical records retrospectively and included only patients with first-ever ischaemic stroke with clinical symptoms.

We defined ischaemic stroke in accordance with the Baltimore-Washington Cooperative Young Stroke Study criteria as an acute onset of focal neurological deficit lasting $>24$ hours, or $<24$ hours with corresponding imaging evidence. ${ }^{18}$ Transient ischaemic attack (TIA) was defined as acute onset of focal neurological deficit lasting < 24 hours, with normal imaging. Patients were excluded if they had TIA, cerebral venous thrombosis, iatrogenic stroke secondary to a procedure or operation, haemorrhage, head or neck trauma, or a diagnosis other than stroke.

Risk factors were investigated in accordance with the 2006 guidelines from the American Stroke Association. ${ }^{19}$ We matched our risk factor definitions closely with those of the largest and most comprehensive study of young people with ischaemic stroke to date, the Helsinki Young Stroke Registry Study, which allowed us to make valid comparisons. ${ }^{3}$

Stroke aetiology was categorised by subtype according to the Trial of Org 10172 in Acute Stroke Treatment (TOAST) criteria. $^{20}$ Subtypes were assigned by two independent stroke neurologists, with the intervention of a third stroke neurologist in the case of a split decision. To allow comparison with previous published studies, both patent foramen ovale (PFO), with or without atrial septal aneurysm (ASA), and blood clotting abnormalities were classified as stroke aetiologies if the clinical setting was consistent and no other source was identified.

An independent neuroradiologist who was blinded to the original diagnosis classified the vascular territory involved and the stroke location. Vascular territory was classified as anterior circulation, posterior circulation, or both. Stroke location was lateral- 
ised and classified as cerebral hemisphere, cerebellum, brainstem, or involving multiple sites.

\section{Statistical analysis}

Categorical variables were compared using the Pearson $\chi^{2}$ test $(P<0.05$ significant). Significant differences between categorical variables for different ethnic subgroups were confirmed using the Fisher exact test.

\section{Results}

Of 666 patients admitted to one of the public hospitals in Adelaide with a primary diagnosis of any form of stroke from January 2006 to June 2010, 326 patients were classified as having had a first-ever occurrence of ischaemic stroke with clinical symptoms.

All patients had had a history taken and had undergone physical examination. Brain imaging had been performed on 325 patients (nearly 100\%), vascular imaging of the neck on 299 patients (92\%), and cardiac imaging on 252 patients (77\%) (Box 1).

The mean age of patients in the study population was 40.7 years (SD, 7.9 years). To assess age-related trends, we divided the population into two groups of equal sizes: a younger cohort aged 15-42 years (162 patients) and an older cohort aged 43-50 years (164 patients). Overall, $56 \%$ of patients (184) were male $(P=0.02)$. The ratio of male to female patients was not significantly different in the younger cohort $(P=0.7)$, but in the older cohort $61 \%$ of patients $(100)$ were men $(P=0.005)$. People of European ancestry formed the largest ethnic subgroup (289; 89\%), and Australian Aboriginals the next largest (19; 6\%).

Overall, the most frequent stroke risk factors were dyslipidaemia (57\%), smoking (49\%) and hypertension (32\%) (Box 2). Obesity (28\%) and illicit drug use $(16 \%)$ were the fourth and eighth most prevalent stroke risk factors, respectively. The most commonly used illicit drug was marijuana (36 patients; $71 \%$ of illicit drug users), followed by amphetamines (19; 37\%). Of amphetamine users, 17 (89\%) administered the drug intravenously. Illicit drug use $(P=0.03)$, PFO $(P<0.001)$, and use of the oral contraceptive pill (OCP; $P=0.005$ ) were more prevalent in the younger cohort, whereas hyper-

1 Methods used to aid diagnosis among patients admitted to public hospitals in South Australia with a primary diagnosis of first-ever ischaemic stroke, Jan 2006 - Jun 2010

Category of diagnostic imaging or test No. of patients $(n=326)$ Specific type of imaging or test (no. [\%])

\begin{tabular}{|c|c|c|}
\hline Brain imaging & 325 (nearly 100\%) & CT, 291 (89\%); MRI, 259 (79\%) \\
\hline Vascular imaging of the neck & 299 (92\%) & $\begin{array}{l}\text { Carotid ultrasound, } 108 \text { (33\%); CTA, } 159 \text { (49\%); MRA, } \\
179(55 \%)\end{array}$ \\
\hline Cardiac imaging & $252(77 \%)$ & TTE, 221 (68\%); TEE, 97 (30\%); cardiac MRI, 15 (5\%) \\
\hline Blood testing for clotting abnormalities & Complete:* 103 (32\%) & Partial (one test or more*): 216 (66\%) \\
\hline
\end{tabular}

testing for clotting abnormalities Complete:* $103(32 \%) \quad$ Partial (one test or more*): 216 (66\%)

* Test for factor V Leiden and prothrombin gene mutations, antithrombin III deficiency, lupus anticoagulant, and anticardiolipin antibody. $\mathrm{CT}=$ computed tomography. MRI = magnetic resonance imaging. CTA = computed tomography angiography. MRA = magnetic resonance angiography. TTE $=$ transthoracic echocardiogram. TEE = transoesophageal echocardiogram.

tension $(P=0.006)$ and diabetes $(P=$ $0.02)$ were more prevalent in the older cohort. More males were heavy alcohol drinkers $(P<0.001)$, and females had more migraines $(P<0.001)$. Cardiovascular disease $(P=0.01)$ and diabetes $(P<0.001)$ were more common among Australian Aboriginals compared with patients of European ancestry.

The most frequently identified stroke aetiology among our study population was cardioembolism (26\%) (Box 3). Arterial dissection, a subtype of the "other aetiology" category, was identified in $15 \%$ of patients (49); and small-vessel occlusion was the aetiology in 10\% (Box 3). Small vessel occlusion was more prevalent in male patients $(P=0.02)$. The most common aetiologies among the 85 patients with cardioembolic stroke were PFO (30), PFO plus ASA (11), infective endocarditis (7), left ventricle thrombus (6) and hypokinetic left ventricle segment (5). Intravenous amphetamine misuse was more common among patients with infective endocarditis $(2 / 7 ; 29 \%)$ compared with the rest of the study population $(15 / 319 ; 5 \%)(P=0.04)$.

Strokes more frequently involved the anterior circulation compared with the posterior circulation $(57 \% \mathrm{v}$ $28 \%$, respectively; $P<0.001$ ) (Box 4 ). Strokes concurrently involving both vascular territories were observed in $9 \%$ of patients; intravenous amphetamine misuse was more common among patients with this type of stroke $(7 / 29 ; 24 \%)$ compared with the whole study population $(P<0.001)$.

\section{Discussion}

To our knowledge, our study is the first specific analysis of a young population of patients with a first-ever ischaemic stroke in Australia. Our data showed some similarities between the population of patients from Adelaide and comparable groups of patients in other countries, but also some significant differences.

Dyslipidaemia, smoking and hypertension were the most frequent stroke risk factors among patients in our study, consistent with results of previous studies of ischaemic stroke among young people of similar age. ${ }^{3-6}$

Obesity and misuse of marijuana and amphetamines were more prevalent in patients from Adelaide compared with those from other countries. Of our study population, $28 \%$ were obese, compared with $11 \%$ and $5 \%$ among patients in the Finnish and Italian young stroke registry studies, respectively $\left(P<0.001\right.$ for each) ${ }^{3,6}$ Sixteen per cent of our study group reported use of illicit drugs, compared with $1 \%$ of patients in the Finnish study $(P<0.001) .^{3}$ Most previous studies of young patients with ischaemic stroke have reported low rates of marijuana and amphetamine use, and that cocaine was the most commonly used illicit drug. ${ }^{21}$ Among our study population, marijuana and amphetamines were the most frequently used illicit drugs, and only one person used cocaine. Marijuana is the illicit drug most often used in South Australia; $12.5 \%$ of South Australians aged 14 years and over have used it at least once. $^{22}$ Evidence supporting marijuana as a stroke risk factor is increasing. Recent studies have shown marijuana users to have increased levels of apolipoprotein C-III, a known cardiovascular risk factor, as well as an adjusted odds ratio of 1.76 for ischaemic stroke. ${ }^{23,24}$ Amphetamines increase the risk of stroke by several mechanisms, including elevation of blood pressure, vasculitis and cerebral vasospasm, ${ }^{25}$ and are frequently mis- 
2 Stroke risk factors among patients admitted to public hospitals in South Australia with a primary diagnosis of first-ever ischaemic stroke, Jan 2006 Jun 2010 , by age, sex and ethnicity $(n=326)$

\begin{tabular}{|c|c|c|c|c|c|c|c|c|c|c|}
\hline & \multirow[b]{2}{*}{$\begin{array}{c}\text { Total } \\
\text { (no. [\%]) }\end{array}$} & \multicolumn{2}{|c|}{ Age in years (no. [\%]) } & \multirow[b]{2}{*}{$P$} & \multicolumn{2}{|c|}{ Sex (no. [\%]) } & \multirow[b]{2}{*}{$P$} & \multicolumn{2}{|c|}{ Ethnicity (no. [\%]) } & \multirow[b]{2}{*}{$P$} \\
\hline & & $\begin{array}{c}15-42 \\
(n=162)\end{array}$ & $\begin{array}{c}43-50 \\
(n=164)\end{array}$ & & $\begin{array}{c}\text { Male } \\
(n=184)\end{array}$ & $\begin{array}{l}\text { Female } \\
(n=142)\end{array}$ & & $\begin{array}{c}\text { European } \\
\text { ancestry } \\
(n=289)\end{array}$ & $\begin{array}{c}\text { Australian } \\
\text { Aboriginal } \\
(n=19)\end{array}$ & \\
\hline Dyslipidaemia & $187(57.4 \%)$ & $87(53.7 \%)$ & $100(61.0 \%)$ & 0.4 & $113(61.4 \%)$ & $74(52.1 \%)$ & 0.3 & $164(56.7 \%)$ & $12(63.2 \%)$ & 0.7 \\
\hline Smoking & $161(49.4 \%)$ & $75(46.3 \%)$ & $86(52.4 \%)$ & 0.4 & $100(54.3 \%)$ & $61(43.0 \%)$ & 0.1 & $146(50.5 \%)$ & $10(52.6 \%)$ & 0.9 \\
\hline Hypertension & $105(32.2 \%)$ & $38(23.5 \%)$ & $67(40.9 \%)$ & 0.006 & $64(34.8 \%)$ & $41(28.9 \%)$ & 0.4 & $85(29.4 \%)$ & $9(47.4 \%)$ & 0.2 \\
\hline Obesity & $92(28.2 \%)$ & $39(24.1 \%)$ & $53(32.3 \%)$ & 0.2 & $50(27.2 \%)$ & $42(29.6 \%)$ & 0.7 & $80(27.7 \%)$ & $9(47.4 \%)$ & 0.1 \\
\hline Alcohol & $63(19.3 \%)$ & $27(16.7 \%)$ & $36(22.0 \%)$ & 0.3 & $51(27.7 \%)$ & $12(8.5 \%)$ & $<0.001$ & $60(20.8 \%)$ & $2(10.5 \%)$ & 0.3 \\
\hline Migraine & $59(18.1 \%)$ & $35(21.6 \%)$ & $24(14.6 \%)$ & 0.1 & $17(9.2 \%)$ & $42(29.6 \%)$ & $<0.001$ & $56(19.4 \%)$ & $3(15.8 \%)$ & 0.7 \\
\hline Cardiovascular disease & $53(16.3 \%)$ & $21(13.0 \%)$ & $32(19.5 \%)$ & 0.1 & $36(19.6 \%)$ & $17(12.0 \%)$ & 0.09 & $40(13.8 \%)$ & $7(36.8 \%)$ & 0.01 \\
\hline Illicit drugs & $51(15.6 \%)$ & $33(20.4 \%)$ & $18(11.0 \%)$ & 0.03 & $34(18.5 \%)$ & $17(12.0 \%)$ & 0.1 & $48(16.6 \%)$ & $3(15.8 \%)$ & 0.9 \\
\hline Patent foramen ovale & $49(15.0 \%)$ & $37(22.8 \%)$ & $12(7.3 \%)$ & $<0.001$ & $23(12.5 \%)$ & $26(18.3 \%)$ & 0.2 & $45(15.6 \%)$ & $2(10.5 \%)$ & 0.6 \\
\hline Diabetes & $44(13.5 \%)$ & $14(8.6 \%)$ & $30(18.3 \%)$ & 0.02 & $24(13.0 \%)$ & $20(14.1 \%)$ & 0.8 & $32(11.1 \%)$ & $9(47.4 \%)$ & $<0.001$ \\
\hline Transient ischaemic attack & $35(10.7 \%)$ & $12(7.4 \%)$ & $23(14.0 \%)$ & 0.07 & $21(11.4 \%)$ & $14(9.9 \%)$ & 0.7 & $31(10.7 \%)$ & $4(21.1 \%)$ & 0.2 \\
\hline Family history & $32(9.8 \%)$ & $12(7.4 \%)$ & $20(12.2 \%)$ & 0.2 & $20(10.9 \%)$ & $12(8.5 \%)$ & 0.5 & $27(9.3 \%)$ & $2(10.5 \%)$ & 0.9 \\
\hline Acute infection & $29(8.9 \%)$ & $15(9.3 \%)$ & $14(8.5 \%)$ & 0.8 & $16(8.7 \%)$ & $13(9.2 \%)$ & 0.9 & $24(8.3 \%)$ & $3(15.8 \%)$ & 0.3 \\
\hline Oral contraceptive pill & $29(8.9 \%)$ & $22(13.6 \%)$ & $7(4.3 \%)$ & 0.005 & na & $29(20.4 \%)$ & - & $26(9.0 \%)$ & $0(0.0 \%)$ & 0.2 \\
\hline Obstructive/central sleep apnoea & $18(5.5 \%)$ & $6(3.7 \%)$ & $12(7.3 \%)$ & 0.2 & $13(7.1 \%)$ & $5(3.5 \%)$ & 0.2 & $16(5.5 \%)$ & $1(5.3 \%)$ & 1 \\
\hline Atrial fibrillation & $15(4.6 \%)$ & $5(3.1 \%)$ & $10(6.1 \%)$ & 0.2 & $8(4.3 \%)$ & $7(4.9 \%)$ & 0.8 & $12(4.2 \%)$ & $2(10.5 \%)$ & 0.2 \\
\hline Factor $\vee$ Leiden mutation & $15(4.6 \%)$ & $7(4.3 \%)$ & $8(4.9 \%)$ & 0.8 & $5(2.7 \%)$ & $10(7.0 \%)$ & 0.07 & $14(4.8 \%)$ & $1(5.3 \%)$ & 0.9 \\
\hline Lupus anticoagulant & $13(4.0 \%)$ & $7(4.3 \%)$ & $6(3.7 \%)$ & 0.8 & $7(3.8 \%)$ & $6(4.2 \%)$ & 0.9 & $12(4.2 \%)$ & $1(5.3 \%)$ & 0.8 \\
\hline Anticardiolipin antibody & $13(4.0 \%)$ & $9(5.6 \%)$ & $4(2.4 \%)$ & 0.2 & $9(4.9 \%)$ & $4(2.8 \%)$ & 0.4 & $11(3.8 \%)$ & $1(5.3 \%)$ & 0.8 \\
\hline High homocysteine & $13(4.0 \%)$ & $7(4.3 \%)$ & $6(3.7 \%)$ & 0.8 & $10(5.4 \%)$ & $3(2.1 \%)$ & 0.1 & $12(4.2 \%)$ & $1(5.3 \%)$ & 0.8 \\
\hline Atrial septal aneurysm & $12(3.7 \%)$ & $7(4.3 \%)$ & $5(3.0 \%)$ & 0.5 & $3(1.6 \%)$ & $9(6.3 \%)$ & 0.03 & $11(3.8 \%)$ & $1(5.3 \%)$ & 0.8 \\
\hline Chronic renal failure & $9(2.8 \%)$ & $3(1.9 \%)$ & $6(3.7 \%)$ & 0.3 & $5(2.7 \%)$ & $4(2.8 \%)$ & 1 & $7(2.4 \%)$ & $2(10.5 \%)$ & 0.05 \\
\hline Active malignancy & $7(2.1 \%)$ & $3(1.9 \%)$ & $4(2.4 \%)$ & 0.7 & $4(2.2 \%)$ & $3(2.1 \%)$ & 1 & $7(2.4 \%)$ & 0 & 0.5 \\
\hline Prothrombin mutation & $6(1.8 \%)$ & $5(3.1 \%)$ & $1(0.6 \%)$ & 0.1 & $4(2.2 \%)$ & $2(1.4 \%)$ & 0.6 & $6(2.1 \%)$ & 0 & 0.5 \\
\hline Protein S deficiency & $4(1.2 \%)$ & $2(1.2 \%)$ & $2(1.2 \%)$ & 1 & $1(0.5 \%)$ & $3(2.1 \%)$ & 0.2 & $4(1.4 \%)$ & 0 & 0.6 \\
\hline Protein C deficiency & $3(0.9 \%)$ & $1(0.6 \%)$ & $2(1.2 \%)$ & 0.6 & $1(0.5 \%)$ & $2(1.4 \%)$ & 0.4 & $1(0.3 \%)$ & 0 & 0.8 \\
\hline Antithrombin III deficiency & $3(0.9 \%)$ & $1(0.6 \%)$ & $2(1.2 \%)$ & 0.6 & $1(0.5 \%)$ & $2(1.4 \%)$ & 0.4 & $3(1.0 \%)$ & 0 & 0.7 \\
\hline Hormone replacement therapy & $1(0.3 \%)$ & 0 & $1(0.6 \%)$ & 0.3 & na & $1(0.7 \%)$ & - & $1(0.3 \%)$ & 0 & 0.8 \\
\hline $\begin{array}{l}\text { na = not applicable. } \\
\text { Definitions for stroke risk factors: } \\
\text { Dyslipidaemia: Total cholesterol, } \geqslant 5.0 \\
\geqslant 3.0 \text { mmol/L; or high-density lipopro } \\
\text { Smoking: Smoked } \geqslant 1 \text { cigarette daily } \\
\text { Hypertension: On antihypertensive the } \\
\text { Obesity: : Ody mass index, } \geqslant 30 \text { or clea } \\
\text { Alcohol: Weekly intake of } \geqslant 200 \mathrm{~g} \text { of a } \\
\text { Migraine: Clearly stated. } \\
\text { Cardiovascular disease: Ischaemic hea } \\
\text { < } 55 \% \text { ), previous myocardial infarctio } \\
\text { Illicit drugs: Taken within the month be } \\
\text { Patent foramen ovale: As reported on } \\
\text { Diabetes: Fasting plasma glucose, } \geqslant 7 \text {. } \\
\text { Transient ischaemic attack: Acute onse } \\
\text { with normal imaging. } \\
\text { Family history: Stroke in a first-degree } \\
\text { Acute infection: Confirmed at stroke or }\end{array}$ & $\begin{array}{l}\text { mmol/L; low-d } \\
\text { tein cholesterol, } \\
\text { uring the previo } \\
\text { erapy, or clearly } \\
\text { arly stated as ob } \\
\text { lcohol. } \\
\text { irt disease, heart } \\
\text { n, or peripheral } \\
\text { efore stroke occ } \\
\text { echocardiogram } \\
\text { O or clearly stat } \\
\text { et of focal neuro } \\
\text { relative. } \\
\text { nset. }\end{array}$ & $\begin{array}{l}<1.0 \mathrm{mmol} / \mathrm{L} \text {. } \\
\text { us year. } \\
\text { tated. } \\
\text { ese. } \\
\text { failure (ejection } \\
\text { /ascular disease } \\
\text { urred. } \\
\text { log (type } 1 \text { or 2). } \\
\text { logical deficit la }\end{array}$ & $\begin{array}{l}\text { In cholesterol, } \\
\text { sting < } 24 \text { hours, }\end{array}$ & $\begin{array}{l}\text { Oral cont } \\
\text { onset. } \\
\text { Obstructi } \\
\text { study. } \\
\text { Atrial fibri } \\
\text { Factor V L } \\
\text { Lupus ant } \\
\text { ratio, > 1.1. } \\
\text { Anticardic } \\
\text { units). } \\
\text { High hom } \\
\text { Atrial sep } \\
\text { Chronic re } \\
\text { Prothrom } \\
\text { Active ma } \\
\text { Protein S, } \\
\text { or warfari } \\
\text { Hormone }\end{array}$ & $\begin{array}{l}\text { aceptive pill: Oe } \\
\text { e or central slee, } \\
\text { llation: Previousl } \\
\text { eiden mutation: } \\
\text { cooagulant: Lupu } \\
\text { 2. } \\
\text { lipin antibody: A } \\
\text { ocysteine: Homo } \\
\text { al aneurysm: As } \\
\text { nal failure: Glom } \\
\text { iin mutation: He } \\
\text { lignancy: Diagno } \\
\text { protein C, antith } \\
\text { n. } \\
\text { replacement the }\end{array}$ & $\begin{array}{l}\text { trogen-based } \\
\text { papnoea: Confi } \\
\text { yor newly diagr } \\
\text { seterozygous. } \\
\text { anticoagulant } \\
\text { nticardiolipin ar }\end{array}$ & $\begin{array}{l}\text { oral contra } \\
\text { irmed obst } \\
\text { nosed; chro } \\
\text { t positive ( } \\
\text { intibody po } \\
\text { umol/L. } \\
\text { hocardiogr }\end{array}$ & $\begin{array}{l}\text { eptive pill beir } \\
\text { Jctive or centr } \\
\text { nic or paroxysr } \\
\text { RVVT [dilute } \\
\text { itive (IgG, } \geqslant 9 \\
\mathrm{~m} \text {. } \\
\mathrm{nl} / \mathrm{min} \text { for }>3\end{array}$ & $\begin{array}{l}\text { aken at tim } \\
\text { leep apnoe } \\
\text { sell's viper } \\
\text { L [lgG phos }\end{array}$ & $\begin{array}{l}\text { stroke } \\
\text { sleep } \\
\text { m test] } \\
\text { lipid] }\end{array}$ \\
\hline
\end{tabular}

used in Australia. ${ }^{22}$ They have been used by $3 \%$ of South Australians aged 14 years and over. ${ }^{22}$

Our analysis of risk factors by age and sex revealed an increase in traditional risk factors with age, more excessive use of alcohol among males, and more migraines among females. These results are similar to results from previous studies. ${ }^{3,6}$ Comparisons by ethnic subgroup revealed a higher prevalence of cardiovascular disease and diabetes among patients who were Australian Aboriginals compared with those of European ancestry - the contrast was particularly striking for diabetes (47\% v 11\%, respectively). Australian Aboriginals were over-represented in our study population, constituting $6 \%$ - more than double the proportion of Aboriginal and Torres Strait Islander people among the Australian population in 2006 (2.3\%). ${ }^{26}$ Comparisons between ethnic subgroups should be interpreted with caution, as the absolute number of Australian Aboriginals included in our study was small. Further studies involving greater numbers of Australian Aboriginals from more areas of Australia are needed.

Cardioembolism, arterial dissection, and small-vessel occlusion were the most frequent stroke aetiologies in our study. The prevalence of cardioembolism was higher among the pop- 
3 Stroke aetiologies among patients admitted to public hospitals in South Australia with a primary diagnosis of first-ever ischaemic stroke, Jan 2006 Jun 2010, by age, sex and ethnicity

\begin{tabular}{|c|c|c|c|c|c|c|c|c|c|c|}
\hline & \multirow[b]{2}{*}{$\begin{array}{c}\text { Total } \\
(n=326)\end{array}$} & \multicolumn{2}{|c|}{ Age in years (no. [\%]) } & \multirow[b]{2}{*}{$P$} & \multicolumn{2}{|c|}{ Sex (no. [\%]) } & \multirow[b]{2}{*}{$P$} & \multicolumn{2}{|c|}{ Ethnicity* (no. [\%]) } & \multirow[b]{2}{*}{$P$} \\
\hline & & $\begin{array}{c}15-42 \\
(n=162)\end{array}$ & $\begin{array}{c}43-50 \\
(n=164)\end{array}$ & & $\begin{array}{c}\text { Male } \\
(n=184)\end{array}$ & $\begin{array}{l}\text { Female } \\
(n=142)\end{array}$ & & $\begin{array}{c}\text { European } \\
\text { ancestry }(n=289)\end{array}$ & $\begin{array}{c}\text { Australian } \\
\text { Aboriginal }(n=19)\end{array}$ & \\
\hline Cardioembolism & $85(26.1 \%)$ & $50(30.9 \%)$ & $35(21.3 \%)$ & 0.09 & $50(27.2 \%)$ & $35(24.6 \%)$ & 0.7 & $70(24.2 \%)$ & $9(47.4 \%)$ & 0.05 \\
\hline Large artery atherosclerosis & $12(3.7 \%)$ & $4(2.5 \%)$ & $8(4.9 \%)$ & 0.3 & $8(4.3 \%)$ & $4(2.8 \%)$ & 0.5 & $11(3.8 \%)$ & 0 & 0.4 \\
\hline Small vessel occlusion & $31(9.5 \%)$ & $12(7.4 \%)$ & $19(11.6 \%)$ & 0.2 & $24(13.0 \%)$ & $7(4.9 \%)$ & 0.02 & $31(10.7 \%)$ & 0 & 0.2 \\
\hline Other determined aetiology & $95(29.1 \%)$ & $46(28.4 \%)$ & 49 (29.9\%) & 0.8 & $48(26.1 \%)$ & $47(33.1 \%)$ & 0.2 & $87(30.1 \%)$ & $1(5.3 \%)$ & 0.05 \\
\hline Multiple possible aetiologies & $25(7.7 \%)$ & $15(9.3 \%)$ & $10(6.1 \%)$ & 0.3 & $15(8.2 \%)$ & $10(7.0 \%)$ & 0.7 & $23(8.0 \%)$ & $2(10.5 \%)$ & 0.7 \\
\hline $\begin{array}{l}\text { Undetermined aetiology, } \\
\text { extensive evaluation }\end{array}$ & $52(16.0 \%)$ & $25(15.4 \%)$ & $27(16.5 \%)$ & 0.8 & $25(13.6 \%)$ & $27(19.0 \%)$ & 0.2 & $44(15.2 \%)$ & $5(26.3 \%)$ & 0.2 \\
\hline $\begin{array}{l}\text { Undetermined aetiology, } \\
\text { incomplete evaluation }\end{array}$ & $26(8.0 \%)$ & $10(6.2 \%)$ & $16(9.8 \%)$ & 0.3 & $14(7.6 \%)$ & $12(8.5 \%)$ & 0.8 & $23(8.0 \%)$ & $2(10.5 \%)$ & 0.7 \\
\hline
\end{tabular}

4 Features seen on neuroimaging among patients admitted to public hospitals in South Australia with a primary diagnosis of first-ever ischaemic stroke, Jan 2006 - Jun2010, by age, sex and ethnicity

\begin{tabular}{|c|c|c|c|c|c|c|c|c|c|c|}
\hline & \multirow[b]{2}{*}{$\begin{array}{c}\text { Total } \\
(n=326)\end{array}$} & \multicolumn{2}{|c|}{ Age in years (no. [\%]) } & \multirow[b]{2}{*}{$P$} & \multicolumn{2}{|c|}{ Sex (no. [\%]) } & \multirow[b]{2}{*}{$P$} & \multicolumn{2}{|c|}{ Ethnicity (no. [\%]) } & \multirow[b]{2}{*}{$P$} \\
\hline & & $\begin{array}{c}15-42 \\
(n=162)\end{array}$ & $\begin{array}{c}43-50 \\
(n=164)\end{array}$ & & $\begin{array}{c}\text { Male } \\
(n=184)\end{array}$ & $\begin{array}{l}\text { Female } \\
(n=142)\end{array}$ & & $\begin{array}{l}\text { European ancestry } \\
\qquad(n=289)\end{array}$ & $\begin{array}{l}\text { Australian Aboriginal } \\
\qquad(n=19)\end{array}$ & \\
\hline \multicolumn{11}{|l|}{ Vascular territory } \\
\hline Anterior circulation & $185(56.7 \%)$ & $96(59.3 \%)$ & $89(54.3 \%)$ & 0.5 & $96(52.2 \%)$ & $89(62.7 \%)$ & 0.2 & $161(55.7 \%)$ & $14(73.7 \%)$ & 0.3 \\
\hline Posterior circulation & $92(28.2 \%)$ & $44(27.2 \%)$ & $48(29.3 \%)$ & 0.7 & $61(33.2 \%)$ & $31(21.8 \%)$ & 0.06 & $86(29.8 \%)$ & $1(5.3 \%)$ & 0.05 \\
\hline Both & $29(8.9 \%)$ & $14(8.6 \%)$ & $15(9.1 \%)$ & 0.9 & $16(8.7 \%)$ & $13(9.2 \%)$ & 0.9 & $23(8.0 \%)$ & $3(15.8 \%)$ & 0.3 \\
\hline \multicolumn{11}{|l|}{ Location } \\
\hline Right CH & $141(43.3 \%)$ & $78(48.1 \%)$ & $63(38.4 \%)$ & 0.2 & $76(41.3 \%)$ & $65(45.8 \%)$ & 0.5 & $124(42.9 \%)$ & $8(42.1 \%)$ & 1 \\
\hline Left $\mathrm{CH}$ & $161(49.4 \%)$ & $79(48.8 \%)$ & $82(50.0 \%)$ & 0.9 & $94(51.1 \%)$ & $67(47.2 \%)$ & 0.6 & $140(48.4 . \%)$ & $12(63.2 \%)$ & 0.4 \\
\hline Right cerebellum & $28(8.6 \%)$ & $11(6.8 \%)$ & $17(10.4 \%)$ & 0.3 & $19(10.3 \%)$ & $9(6.3 \%)$ & 0.2 & $24(8.3 \%)$ & $1(5.3 \%)$ & 0.7 \\
\hline Left cerebellum & $29(8.9 \%)$ & $14(8.6 \%)$ & $15(9.1 \%)$ & 0.9 & $18(9.8 \%)$ & $11(7.7 \%)$ & 0.5 & $21(7.3 \%)$ & $2(10.5 \%)$ & 0.6 \\
\hline Right BS & $19(5.8 \%)$ & $8(4.9 \%)$ & 11 (6.7\%) & 0.5 & $12(6.5 \%)$ & $7(4.9 \%)$ & 0.6 & $18(6.2 \%)$ & $1(5.3 \%)$ & 0.9 \\
\hline Left BS & $23(7.1 \%)$ & $8(4.9 \%)$ & 15 (9.1\%) & 0.2 & 18 (9.8\%) & $5(3.5 \%)$ & 0.04 & $21(7.3 \%)$ & $1(5.3 \%)$ & 0.8 \\
\hline Multiple sites & $61(18.7 \%)$ & 31 (19.1\%) & $30(18.3 \%)$ & 0.9 & $27(14.7 \%)$ & 34 (23.9\%) & 0.06 & $54(18.7 \%)$ & $3(15.8 \%)$ & 0.8 \\
\hline
\end{tabular}

ulation in Adelaide (26\%) than among patients in Finland (20\%), Greece (13\%), Spain (17\%), Canada $(14 \%)$, Indiana in the United States $(14 \%)$, and Taiwan $(18 \%),{ }^{3-5,9,10,14}$ but not significantly different compared with Georgia, Italy, Sweden, Switzerland, Iowa in the US, Brazil, India and Korea. ${ }^{3-16}$ Infective endocarditis was more frequent among our study population than has been reported in most other studies. ${ }^{3,13,14}$ Since intravenous injection can trigger infective endocarditis, our higher observed rates of cardioembolic stroke might be partly explained by a high prevalence of intravenous amphetamine misuse.

The few studies that have reported ischaemic stroke topography among young adults have shown that stroke involving the anterior cerebral circulation are more frequent than those involving the posterior circulation, observations which are consistent with our results. 3,7,17 However, more strokes in South Australia (9\%) concurrently involved both the anterior and the posterior circulations compared with Finland (5\%) and Sweden (3\%) $\left(P<0.05\right.$ for each). ${ }^{3,7}$ Intravenous amphetamines have the potential to cause infarction in more than one vascular territory simultaneously, due to their pathophysiological effects. Such imaging findings in our study are thus probably partly explained by a high prevalence of intravenous amphetamine misuse among patients with this type of stroke.

There were several limitations to our study. First, it was retrospective in nature. It covered a period of several years over which imaging technology and diagnostic testing varied. It also involved several hospitals, so the approach to documentation and diagnostic testing was not standardised. The retrospective determination of risk factors is of particular concern in that the more atypical risk factors (such as illicit drug use) may have been underestimated, as they are not routine components of history-taking. Second, the Australian health care system comprises coexisting public and private sectors, and access to patient details in the private system is difficult. We were unable to include private patients in our study. However, the actual number of younger patients admitted to private hospitals with ischaemic stroke during our study period was low - roughly seven patients per year, before applying exclusion criteria (South Australian Department of Health, unpublished data, 2010), and it is therefore unlikely that these patients would have 
affected our results significantly. Third, some younger patients with ischaemic stroke treated solely as outpatients may have been missed by our study. However, it is hard to imagine that there would be many patients in this group, as young patients with a first-ever ischaemic stroke with clinical symptoms would routinely be admitted to one of the tertiary hospitals included in our study for inpatient workup. Finally, since risk factor definitions vary from one study to the next, any comparison of risk factors across different studies must be interpreted with caution. Although we matched our risk factor definitions with those of the Helsinki study, any comparisons with other studies of ischaemic stroke in young people are subject to this limitation.

Our findings highlight the need to educate health care providers, as well as the public in general, about the association between illicit drug use and stroke. There is also a clear need for registries specific to ischaemic stroke in young people that include patients from both the public and private arms of the medical system. Finally, larger prospective epidemiological studies are needed to address this area of growing concern.

Acknowledgements: We thank our statistician, John Field. We also thank the South Australian Department of Health for providing data on private patient numbers.

Competing interests: No relevant disclosures.

Received 3 May 2011, accepted 27 Jul 2011.

1 Australian Institute of Health and Welfare and National Heart Foundation of Australia. Heart, stroke and vascular diseases - Australian facts 2004. Canberra: AlHW and National Heart Foundation of Australia, 2004. (AlHW Cat. No.
CVD 27.) http://www.aihw.gov.au/publications/ cvd/hsvd04/hsvd04.pdf (accessed Dec 2010).

2 Dewey HM, Thrift AG, Mihalopoulos, et al. Cost of stroke in Australia from a societal perspective: results from the North East Melbourne Stroke Incidence Study (NEMESIS). Stroke 2001; 32: 2409-2416.

3 Putaala J, Metso A, Metso T, et al. Analysis of 1008 consecutive patients aged 15 to 49 with first-ever ischemic stroke: the Helsinki Young Stroke Registry. Stroke 2009; 40: 1195-1203.

4 Spengos K, Vemmos KN. Risk factors, etiology, and outcome of first-ever ischemic stroke in young adults aged 15 to 45 - the Athens young stroke registry. Eur J Neurol 2010; 17: 1358-1364.

5 Varona JF, Guerra JM, Bermejo F, et al. Causes of ischemic stroke in young adults, and evolution of the etiological diagnosis over the long term. Eur Neurol 2007: 57: 212-218.

6 Rasura M, Spalloni A, Ferrari M, et al. A case series of young stroke in Rome. Eur J Neurol 2006; 13 : 46-152.

7 Kristensen B, Malm J, Carlberg B, et al. Epidemiology and etiology of ischemic stroke in young adults aged 18 to 44 years in northern Sweden. Stroke 1997; 28: 1702-1709.

8 Nedeltchev K, der Maur TA, Georgiadis D, et al. Ischemic stroke in young adults: predictors of outcome and recurrence. J Neurol Neurosurg Psychiatry 2005; 76: 191-195.

9 Chan MT, Nadareishvili ZG, Norris JW. Diagnostic strategies in young patients with ischemic stroke in Canada. Can J Neurol Sci 2000; 27: 120-124.

10 Williams LS, Garg BP, Cohen M, et al. Subtypes of ischemic stroke in children and young adults. Neurology 1997; 49: 1541-1545.

11 Qureshi Al, Safdar K, Patel M, et al. Stroke in young black patients. Risk factors, subtypes, and prognosis. Stroke 1995; 26: 1995-1998.

12 Adams HP, Kappelle LJ, Biller J, et al. Ischemic stroke in young adults. Experience in 329 patients enrolled in the lowa registry of stroke in young adults. Arch Neurol 1995; 52: 491-495.

13 Siqueira Neto JI, Santos AC, Fabio SR, Sakamoto AC. Cerebral infarction in patients aged 15 to 40 years. Stroke 1996; 27: 2016-2019.

14 Lee TH, Hsu WC, Chen CJ, Chen ST. Etiologic study of young ischemic stroke in Taiwan. Stroke 2002; 33:1950-1955.

15 Lipska K, Sylaja PN, Sarma PS, et al. Risk factors for acute ischemic stroke in young adults in South India. J Neurol Neurosurg Psychiatry 2007; 78: 959-963.
16 Kwon SU, Kim JS, Lee JH, Lee MC. Ischemic stroke in Korean young adults. Acta Neurol Scand 2000; 101: 19-24.

17 Naess H, Nyland HI, Thomassen L, et al. Incidence and short-term outcome of cerebral infarction in young adults in western Norway. Stroke 2002; 33: 2105-2108.

18 Johnson CJ, Kittner SJ, McCarter RJ, et al. Interrater reliability of an etiologic classification of ischemic stroke. Stroke 1995; 26: 46-51.

19 Goldstein LB, Adams R, Alberts MJ, et al; American Heart Association; American Stroke Association Stroke Council. Primary prevention of ischemic stroke: a guideline from the American Heart Association/American Stroke Association Stroke Council: cosponsored by the Atherosclerotic Peripheral Vascular Disease Interdisciplinary Working Group; Cardiovascular Nursing Council; Clinical Cardiology Council; Nutrition, Physical Activity, and Metabolism Council; and the Quality of Care and Outcomes Research Interdisciplinary Working Group. Circulation 2006; 113: e873-e923.

20 Adams HP Jr, Benedixen BH, Kappelle $\sqcup$, et al. Classification of subtype of ischemic stroke. Definitions for use in a multicenter clinical trial. TOAST. Trial of Org 10172 in Acute Stroke Treatment. Stroke 1993; 24: 35-41.

21 Sloan MA, Kittner SJ, Feeser BR, et al. Illicit drugassociated ischemic stroke in the BaltimoreWashington young stroke study. Neurology 1998; 50:1688-1693.

22 Drug and Alcohol Services South Australia. Statistics on illicit drug use in South Australia. Adelaide: SA Health, 2007. http:// www.dassa.sa.gov.au/site/page.cfm?u=204 (accessed Jan 2011).

23 Westover AN, McBride S, Haley RW. Stroke in young adults who abuse amphetamines or cocaine. Arch Gen Psychiatry 2007; 64: 495-502.

24 Jayanthi S, Buie S, Moore S, et al. Heavy marijuana users show increased serum apolipoprotein C-III levels: evidence from proteomic analyses. Mol Psychiatry 2010; 15: $101-112$

25 Petitti DB, Sidney S, Quesenberry C, Bernstein A. Stroke and cocaine or amphetamine use. Epidemiology 1998; 9: 596-600.

26 Australian Bureau of Statistics. 2006 Census QuickStats: Australia. Canberra: ABS, 2006. http://www.censusdata.abs.gov.au/ ABSNavigation/prenav/ViewData?subaction=1\&producttype=QuickStats\&areacode= 0\&action= 401\&collection=Census\&textversion= false\&breadcrumb=PL\& (accessed Jan 2011). 口 